\title{
Cancer's vast secretes revealed-secretome changes promote resistance to therapy
}

$\mathrm{T}$ argeted therapies that disrupt oncogenic signalling pathways are associated with high response rates in populations of patients with tumours harbouring sensitizing mutations; however, the degree of tumour regression and the duration of clinical responses are limited, and development of drug resistance and cancer progression is uniformly observed. Now, a preclinical study by Anna Obenauf et al. has disclosed the secrets of resistance to tyrosine kinase inhibitors (TKIs). These new insights have clinical implications, revealing rational combination therapies that might avert or at least delay resistance.

"The rapid emergence of clinical drug resistance might be facilitated by a small number of pre-existing cancer cells that are intrinsically resistant or poised to quickly adapt to drug treatment," explains Obenauf. Thus, in vitro and in vivo models of heterogeneous tumour cell populations, in which a small population of resistant tumour cells was present at the start of therapy, were developed. "The first step was to establish cell lines resistant to targeted therapy by exposing the drugsensitive parental population to kinase inhibitors in vitro." Obenauf continues, "after several weeks, we retrieved cells that were resistant to higher doses of the drug and transduced them with a construct expressing green fluorescent protein and luciferase, allowing us to track growth and metastasis of the resistant tumourcell population in a variety of animal models using bioluminescent imaging." Tumour heterogeneity was modelled by injecting these labelled, treatment-resistant cells with an excess of unlabelled, drugsensitive cells, at proportions of $0.05 \%$ and $99.95 \%$, respectively.

Using this approach, the growth of A375 human melanoma cell xenografts was examined in mice, in the context of BRAF inhibitor (BRAFi) therapy with vemurafenib. Despite regression of tumours formed from admixtures

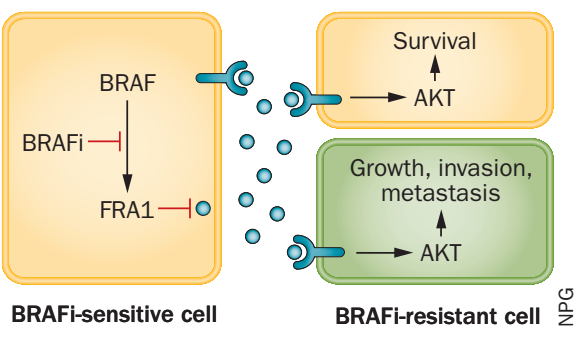

of sensitive and resistant cells during treatment, the BRAFi-resistant cell population expanded and the growth rate of these cells was increased by vemurafenib, compared with vehicle. In addition, locally increased outgrowth of the BRAFi-resistant cell population in regressing tumours was associated with a greater metastatic burden in the lung. Interestingly, tumours formed from only BRAFi-resistant cells did not demonstrate these differences, suggesting that drug-sensitive cells are required to drive proliferation of the resistant cells. Similar findings were reported with other modes of BRAF inhibition, with different and clinically relevant BRAFisensitive/resistant cell admixtures, and for admixtures of cells resistant and sensitive to crizotinib or erlotinib in tumours driven by ALK and EGFR alterations, respectively.

"We also found that the regressing tumours in our animal models acted as potent 'magnets' to attract resistant cells from the circulation, a process called cancer self-seeding," states Joan Massagué, senior author of the study. Indeed, intracardially injected BRAFiresistant clones were recruited to $95 \%$ of vemurafenib-treated regressing tumours, but only $12.5 \%$ of vehicle-treated, BRAFisensitive tumours; seeding of BRAFiresistant clones was shown to accelerate relapse of BRAFi-sensitive tumours. Particularly in metastatic disease, "this phenomenon could add an additional layer of complexity by distributing resistant cancer-cell clones to regressing tumours," Massagué continues.
The authors hypothesized that TKIexposed drug-sensitive cells release soluble mediators that influence drug-resistant clones (and potentially stromal cells) into the tumour microenvironment. This theory was confirmed in vitro. Moreover, the soluble factors were found to suppress apoptosis and thus increase the survival of the drug-sensitive cell lines.

Gene-expression analyses revealed vast, overlapping changes in the levels of proteins secreted by cancer cell lines harbouring different oncogenic drivers, targeted with different TKIs, suggesting the existence of a 'therapy-induced secretome' (TIS). Further experiments implicated downregulation of the ERK effector and transcriptional regulator FRA1 in this TIS response, and this protein was found to be downregulated in biopsies from BRAFitreated patients with melanoma. In situ transcriptome analysis of drug-resistant melanoma cells responding to the TIS in the regressing tumour microenvironment revealed hyperactivation of multiple signalling pathways, most prominently the $\mathrm{PI} 3 \mathrm{~K} / \mathrm{AKT} / \mathrm{mTOR}$ pathway.

Importantly, dual inhibition of the MAPK (BRAF/ERK) and PI3K/AKT/ mTOR pathways attenuated the amplified growth of and tumour seeding by BRAFiresistant cells in the xenograft model in the presence of BRAFi-sensitive cells. "Our results provide a rationale for combining $\mathrm{PI} 3 \mathrm{~K} / \mathrm{AKT} / \mathrm{mTOR}$ pathway inhibitors with inhibitors of the MAPK pathway in the treatment of these tumours," Obenauf concludes. "However, the breadth of the TIS and the generality of our findings suggest the combination of molecularly targeted agents with a radically different therapeutic modality might be required for durable responses," adds Massagué.

\section{David Killock}

Original article Obenauf, A. C. et al. Therapy-induced tumour secretomes promote resistance and tumour progression. Nature doi:10.1038/nature14336 